\title{
Cross Layer Framework for Traffic Management During Vertical Handover in HetNets
}

\author{
Mrs.R.Mohanapriya ${ }^{\# 1}$, Dr.K.B.Jayanthi ${ }^{\# 2}$ \\ ${ }^{\#}$ ECE Department, Paavai Engineering College, Namakkal, India \\ ${ }^{\#}$ ECE Department, KSR College of Technology, Tiruchengode, India \\ ${ }^{1}$ mprmpriya@gmail.com \\ 2jayanthikb@gmail.com
}

\begin{abstract}
Next Generation Heterogeneous Networks uncovers few challenges, which still exist and thwart in providing seamless services during mobility. Heterogeneous networks differ in their capabilities, services and scalability, therefore, to handle handoff mechanism it becomes more complex. It involves the integration of different access network technologies that differ in reliability, bandwidth or cost but intelligent enough to connect the entire world without limits. Handoff initialization and network selection are important phases to be performed well to ensure ideal QoS and better traffic management to customers. In this paper, a cross layer control mechanism is proposed for the execution of vertical handoff in three different networks (Wi-Fi, WiMAX and LTE) with the goal of maximizing the quality of service faced by each user in terms of packet dropping rate, handoff failure rate, and handoff latency. Simulations carried out using Ns-3 and results were obtained.
\end{abstract}

Keyword - Cross layer information, Heterogeneous wireless networks, Vertical Handover, Traffic adaptation.

\section{INTRODUCTION}

The wireless network environment consists of different access technologies, such as Wireless Local Area Network (WLAN), Wireless Interoperability for Microwave Access (WiMAX) and 2G/3G/4G cellular mobile networks. In addition, within access technologies, there are different implementations resulting in different sized cells and features. Together these constitute a heterogeneous network environment, where different base stations (BSs) of different access technologies provide varying characteristics and capabilities. They support simultaneous connectivity to multiple overlapping access networks that may be based on multiple access technologies. For a satisfactory user experience, mobile terminals must be able to seamlessly transfer to the "best" access link among all available candidates with no perceivable interruption to an ongoing conversation which can be a video or voice session. Such ability to handover between heterogeneous networks is referred to as seamless vertical handovers [1].

Whether a handover is mobile device-initiated or network-initiated, finding the most optimal handover target in the range of the mobile device currently requires network scanning in different bands. Potential handover targets may be lacking and then use of the current access will need enhancement. In this case, traffic priority management, user priorities, and sharing the available transmission resources more efficiently for the application data become more momentous factors. Traffic-priority and QoS-class-based packet scheduling schemes have been implemented in basically all novel broadband wireless systems such as LTE [6], WiMAX [5], and WLAN [4].

In this paper three different networks are considered namely WiFi, WiMAX and LTE. LTE or long-term evolution, is a type of mobile broadband that rivals WiMAX Both services are IP-based and use a technology called orthogonal frequency-division multiplexing (OFDM) access. They also use a type of wireless technology that lets people get high-speed Internet across coverage areas that span miles. Table [1] shows the comparison of different parameters of the three different networks [3]. 
Table 1. Different Parameters of WiFi,WiMAX and LTE

\begin{tabular}{|l|l|l|l|}
\hline Parameters & WiFi & WiMAX & LTE \\
\hline Frequency band & $\begin{array}{l}2.4 \mathrm{GHz} \text { to } 5 \mathrm{GHz} \\
\text { (Unlicensed) }\end{array}$ & $\begin{array}{l}2.3 \mathrm{GHz}, 2.5 \mathrm{GHz}, 3.5 \\
\text { GHz, 5.8GHz (Unlicensed) }\end{array}$ & $2 \mathrm{GHz}$ \\
\hline Channel bandwidth & $20 \mathrm{MHz}$ & Up to 20 MHz & Up to 20 MHz \\
\hline Downlink and Uplink & DSSS QPSK & OFDMA OFDMA & OFDMA SC-FDMA \\
\hline Peak data rate & Up to $54 \mathrm{Mbps}$ & $75 \mathrm{Mbps}$ & $100 \mathrm{Mbps}$ \\
\hline Cell capacity & $50-100$ users & $100-200$ users & $\begin{array}{l}>200 \text { users at } 5 \mathrm{MHz} \\
>400 \text { users with } \\
\text { Larger bandwidth }\end{array}$ \\
\hline Mobility Support & Up to $120 \mathrm{~km} / \mathrm{h}$ & Up to $120 \mathrm{~km} / \mathrm{h}$ & Up to $350 \mathrm{~km} / \mathrm{h}$ \\
\hline Cell radius & $150 \mathrm{~m}$ & $2-7 \mathrm{Km}$ & $5 \mathrm{Km}$ \\
\hline
\end{tabular}

\section{Cross LAYER ARChitecture AND INFORMATION SERVICES}

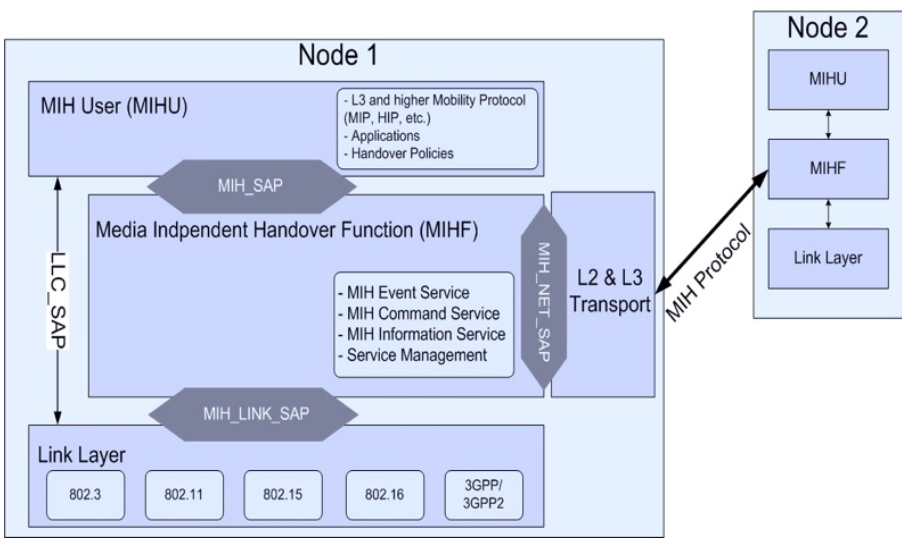

Fig. 1.MIH Architecture

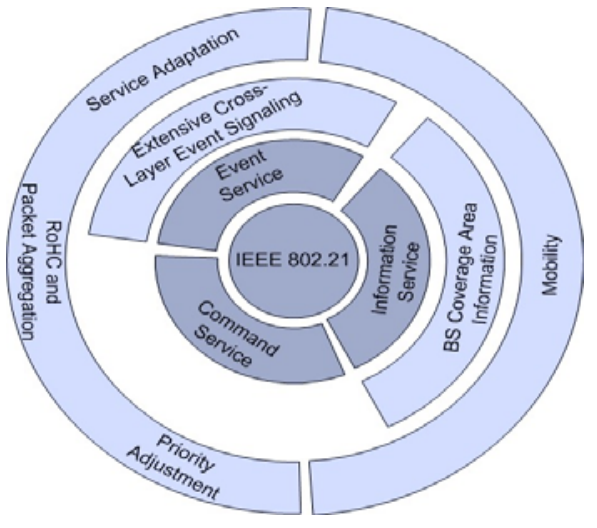

Fig. 2. Cross Layer Information services

Figure 1 represents the Media Independent Handover Architecture. IEEE 802.21 is the first widely cited standard that facilitates mobility between networks based on different access technologies. Enablers of interaccess technology mobility, such as MIP, have been available before IEEE 802.21. Figure 1 and Figure 2 highlights the important services of the Cross Layer Architecture. The three main services of the standard comprise event dissemination, command service, and information service. Media Independent Event Service (MIES) enables event reporting from link layers locally or from remote MIHF entities. Media Independent Command Service (MICS) provides a set of commands for MIHU to manage and control link layer behaviour. Media Independent Information Service (MIIS) allows querying for information about the capabilities of nearby networks and their access points. Service Management allows finding out the remote entities within the current network and carrying out registrations and event subscriptions [13].

IEEE 802.21 supports using quality reports about the link condition as the basis for handover decisions. Triggering Framework-facilitated BS traffic-load-driven mobility management has been studied by various authors [13]. However, they did not monitor the quality of the application used in mobility management, which could result in even better mobility performance.

\section{Proposed System ANd Parameters Evaluation}

Traffic Management is supported by state-of-the-art access technologies such as Wi-Fi, WiMAX and LTE. The QoS classes defined in these technologies are not widely exploited in commercial networks. It is common to use fixed resource allocations for certain application types in the configurations of QoS classes. It is likely that these minimum allocations are not sufficient for all traffic flows. Hence the triggering mechanism of MIH is used in this cross layer framework architecture to reduce the Handover latency and Failure rate. It provides more flexible and extensive signalling capabilities and manages event delivery between event producers and consumers that can reside anywhere in the network. Thus, in order to optimize the benefits QoS classes, adjustment of QoS class definitions needs to be carried out dynamically according to the requirements of current applications. 


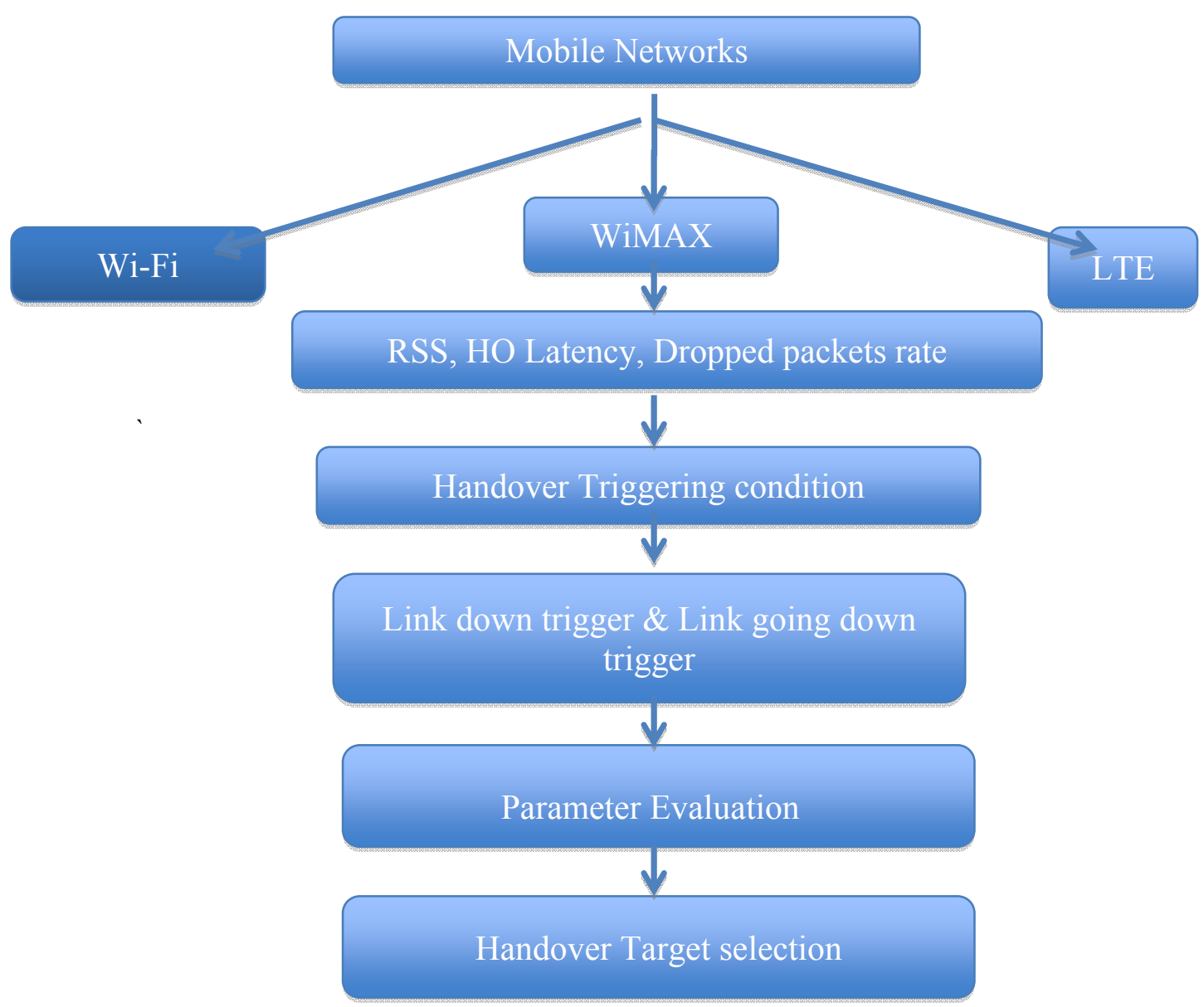

Fig. 3. Proposed System Framework

In the above figure, handover triggering conditions (Link_Goingdown, Link_Down) are analyzed. Whenever a packet is received with RSSI lower than the specified Link_Going _down, then the corresponding event trigger is generated and the event counter is incremented. When the counterequals the Link_Down_Event_threshold then the handover is initiated. When it exceeds the threshold value, the event trigger will be reset, it cancels the current network and switches to a new network. The value of Link_ Going _down and Link_Down_Event_threshold can be optimized to reduce the handover latency and handover failure rate. The Link Going Down threshold values at various mobile speeds are fixed and simulations carried out using NS3. Since there is a performance difference between Wi-Fi, WiMAX and LTE handover, all the three handovers are analyzed. The attained handover latency and the number of dropped packets are presented.

The parameters analyzed are Packet Dropping Rate, Handover Failure Rate and Handover Latency.

Packet Dropping Rate:It is defined as the fraction of the total transmitted packets that did not arrive at the receiver.

Handover Failure Rate: It is dfined as the ratio of number of handover failures to the total number of handover attempts wherein the total number of handover attempts is defined as the sum of the number of handover failures and number of successful handovers

Handover Latency: It is defined as the time duration within which packets are getting dropped due to the handover action.

The following steps are carried out to select the target network for the above-mentioned parameters.

Step 1: Discover the available networks based on Received Signal strength (RSS).

Step 2: Handover Triggering conditions (Link_ going Down and Link__Down) are evaluated.

Step 3:Select the network with optimum performance for various parameters.

Step 4:Trigger the handover

Step 5:Perform make-before-break connection. 


\section{IV.RESULTS AND DISCUSSION}

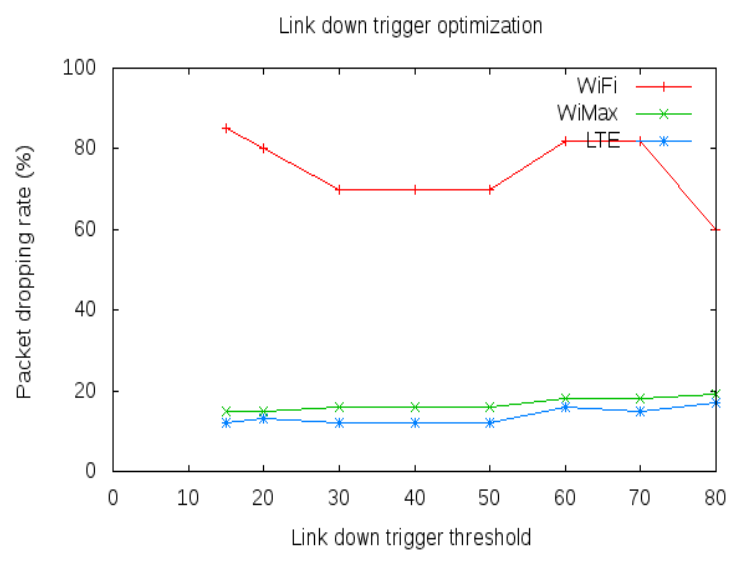

Fig. 4. Packet Dropping Rate

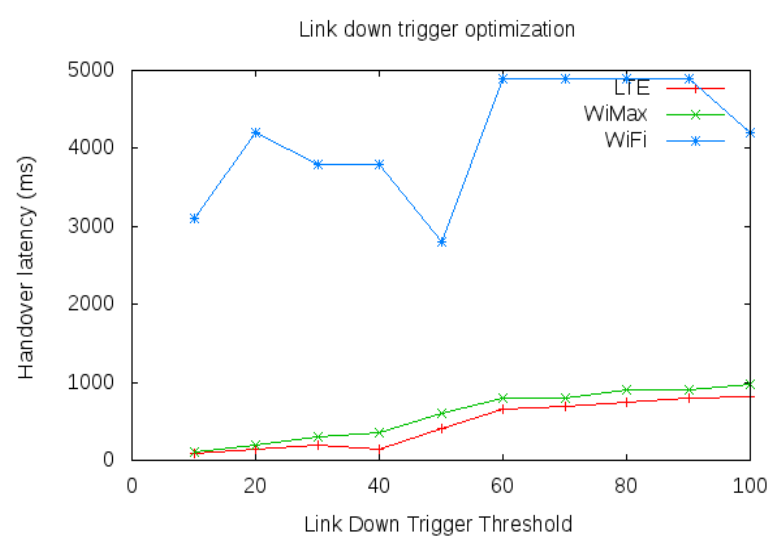

Fig. 5.Handover Latency

From Figure 4 and Figure 5, it is implied that lower values of Link down trigger threshold, the packet dropping rates and Hanover Latency is low for LTE network when compared to WiMAX and WiFi network. As the threshold value increases there are variations for both packet dropping rates and Hanover Latency. Hence the optimum value for Link down trigger threshold can be approximated as $50(\mathrm{dBm})$.

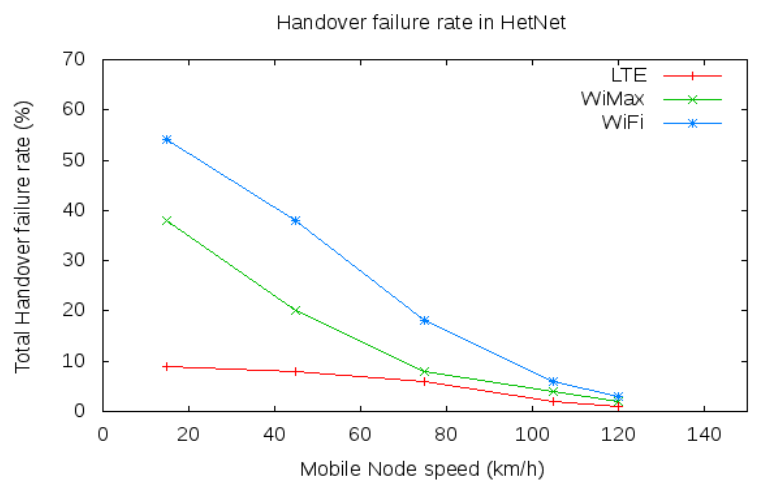

Fig. 6. Handover Failure Rate

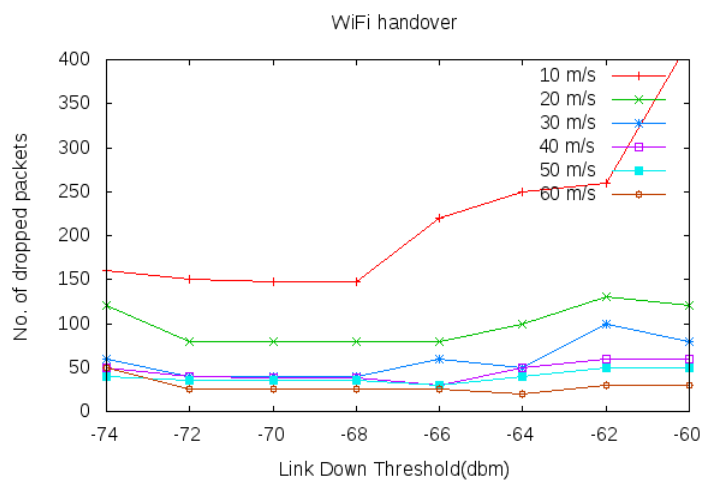

Fig.7.Dropped Packets (WiFi)

Handover at lower speeds impact the performance more when compared to Handover at higher speeds, which is indicated in Figure 6. So the speed and the distance at which the mobile nodes travels plays an important role in reducing the number of handover failures.

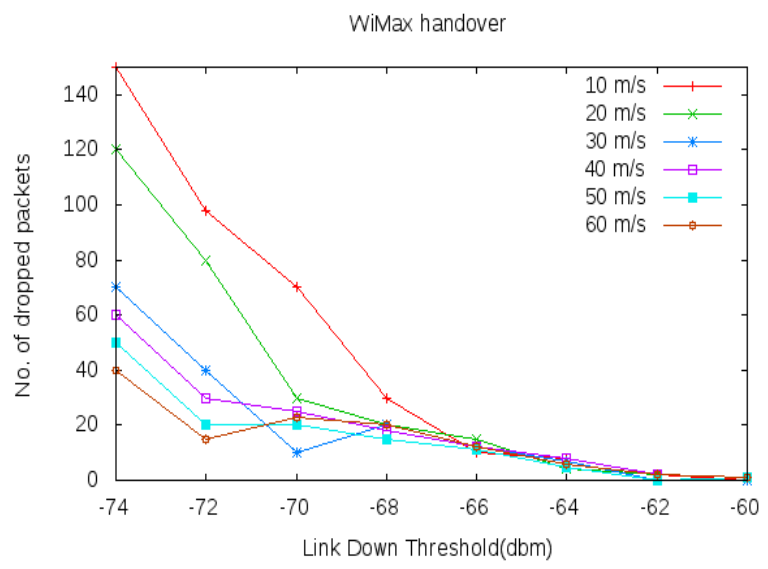

Fig. 8.Dropped Packets (WiMAX)

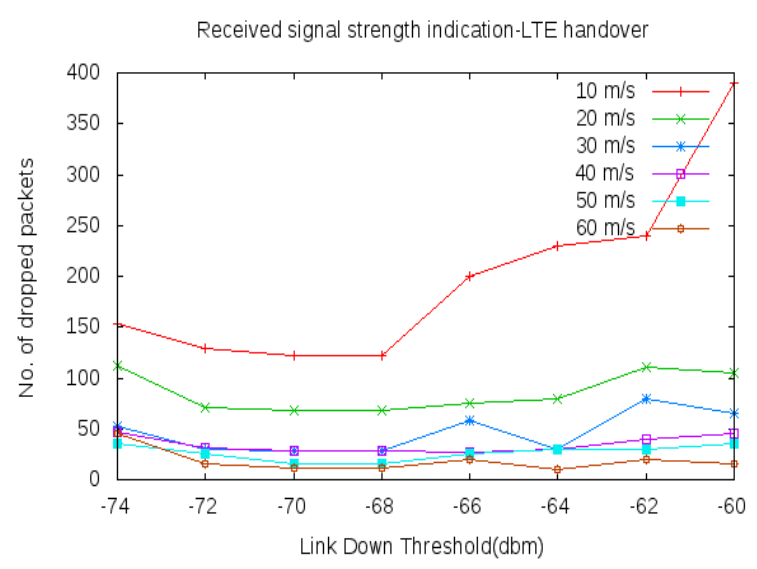

Fig.9.Dropped Packets (LTE) 
Through simulation results it is observed from Figure 7, Figure 8 and Figure 9 that number of dropped packets is more at higher thresholds that is inversely related to the speed. In case of WiFi and LTE networks, at a speed of $60 \mathrm{~m} / \mathrm{s}$, the number of dropped packets is less on comparing with a speed of $10 \mathrm{~m} / \mathrm{s}$ or so. In case of WiMAX network, the number of dropped packets decreases with increasing threshold. So the threshold is a major RSSI indicator for all the three networks.

\section{V.CONCLUSION}

In this paper the triggering conditions of the Cross layer architecture is taken into consideration for the analysis of various traffic parameters. Command services of Media Independent Handover are not analysed in many cases. In future work the command services and comparison with different protocols will be taken into account.

\section{REFERENCES}

[1] W. Mohr and W. Konhauser.Access network evolution beyond third generation mobile communications.IEEE Wireless Communications Magazine, 38(12): 122-133, 2000

[2] Srivastava V \&Motani M (2005) Cross-layer design: A survey and the road ahead. IEEE Communications Magazine 43(12): 112119.

[3] A Survey on Handoffs - Lessons for $60 \mathrm{GHz}$ based Wireless Systems Bien Van Quang, R. Venkatesha Prasad, IgnasNiemegeers Electrical Engineering, Mathematics, and Computer Science Faculty Delft University of Technology .

[4] IEEE (2012). Part 11: Wireless LAN medium access control (MAC) and physical layer (PHY) specifications. Amendment 2: MAC enhancements for robust audio video streaming. IEEE Std 802.11aa-2012.

[5] IEEE (2011).IEEE standard for local and metropolitan area networks. Part 16: Air interface for broadband wireless access systems. Amendment 3: Advanced air interface. IEEE Std 802.16m-2011 (Amendment to IEEE Std 802.16-2009).

[6] 3GPP (2012). Evolved universal terrestrial radio access (E-UTRA); medium access control (MAC) protocol specification (release 8). 3GPP TS 36.321 version 8.12.0.

[7] N. Nasser, A. Hasswa, and H. Hassanein.Handoffs in fourth generation heterogeneous networks. IEEE Communications Magazine, 44(10):96-103, 2006.

[8] B. Chang and J. Chen, "Cross-Layer-Based Adaptive Vertical Handoff With Predictive RSS in Heterogeneous Wireless Networks," IEEE Trans. Vehicular Technology. vol. 57, no. 6, pp. 3679-3692, Nov. 2008.

[9] M.Liu, Z. Li, and E. Dutkiewicz, "Performance Analysis and Optimization of Handoff Algorithms in Heterogeneous Wireless Networks," IEEE Trans. Mobile Comp., vol. 7, no. 7, pp. 846-857, July 2008.

[10] Eastwood Les, Migaldi Scott, Gupta Vivek, Xiayou. Mobility using IEEE 802.21 in heterogeneous IEEE 802.16/802.11-based, IMTadvanced (4G) network. IEEE Wireless Commun 2008;15(2):26-34.

[11] Lampropoulos George, Passas Nikos, MerakosLazaros, Kaloxylos Alexandros. Handover management architectures in integrated WLAN/cellular networks.IEEE Communication Surveys Tutorials 2005; 7(4).

[12] J.Jackson Juliet Roy, V.Vaidehi, M.S.Sricharan' QoS guaranteed integration methodology for a WLAN-WiMAX heterogeneous network, 2011

[13] Piri, Esa, Improving heterogeneous wireless networking with cross-layer information services. University of Oulu Graduate School; University of Oulu, Faculty of Information Technology and Electrical Engineering, Department of Communications Engineering ,Acta Univ. Oul. C 529, 2015

\section{AUTHORS PROFILE}

Mrs.R.Mohanapriya is working as an Assistant Professor in the Department of Electronics and Communication Engineering at Paavai Engineering College. She completed her B.E., in KSR College of Technology and her M.E.(Gold Medallist) in Paavai Engineering College She has over seven years of experience in the teaching field and her interests include Wireless Networking,Wireless Communication .

Dr.K.B.Jayanthi is working as Head of the Department in the Department of Electronics and Communication Engineering at KSR College of Technology,Tiruchengode. She completed her B.E.and M.E in Government College of Engineering and has over 20 years of experience in the teaching field. Her research interests include Medical Image Processing. 